\title{
Evaluation of Cardiovascular System Involvement in Hospitalized Cases of Swine Flu
}

\author{
Bal Kishan Gupta*, Rakesh Kumar Baberwal, Anjli Gupta, Shyam Lal Meena, Narendra Dara, Kashish Narula, Jigyasa Gupta, \\ and Sunil Rulaniya
}

Department of Medicine, Sardar Patel Medical College and PBM Hospital, Bikaner, Rajasthan, India

*Corresponding author: Bal Kishan Gupta, Senior Professor, In-charge Medical ICU, Department of Medicine, Sardar Patel Medical College and PBM Hospital, Bikaner-334003, Rajasthan, India, Tel: +91151 200218, +919829176143; Fax: +91151 2226301; E-mail: bkgbkn@rediffmail.com

Received: 15 Mar, 2020 | Accepted: 01 Apr, 2020 | Published: 07 Apr, 2020

Citation: Gupta BK, Baberwal RK, Gupta A, Meena SL, Dara N, et al. (2020) Evaluation of Cardiovascular System Involvement in Hospitalized Cases of Swine Flu. J Emerg Dis Virol 5(1): dx.doi.org/10.16966/2473-1846.149

Copyright: (c) 2020 Gupta BK, et al. This is an open-access article distributed under the terms of the Creative Commons Attribution License, which permits unrestricted use, distribution, and reproduction in any medium, provided the original author and source are credited.

\begin{abstract}
Introduction: Swine flu primarily affects respiratory system but there are case reports on involvement of cardiovascular system.

Materials and Methods: A total of 79 cases of swine flu confirmed by RT-PCR admitted in tertiary care hospital were studied. Cardiovascular involvement was evaluated by clinical history and examination, Standard 12 lead ECG, Chest X-Ray, CPK-MB, NT-proBNP and Troponin I. Other laboratory investigations like complete blood count, renal function test, liver function test, blood sugar, CRP titre, and ABG were also done. All patients were treated as per WHO guidelines and followed-up during hospital stay.

Results: There were 35 (44.3\%) males (mean age $52.89 \pm 17.7$ and 44 (55.7\%) females (mean age $47.36 \pm 16.79$ ). 49 Cases belong to category C and 15 cases each in category B1 and B2. 13 cases (16.46\%) had one or more underlying pre-existing cardiovascular disease (PCVD). Cardiovascular manifestations at the time of admission were breathlessness $(72.2 \%)$, chest pain $(28.8 \%)$, palpitation (16.5\%), presyncope (10.1\%), syncope $(7.6 \%)$ and giddiness (5.1\%). ECG manifestation were prolonged QTc interval, RBBB, LBBB, AF, 1ST degree AV block and ST segment elevation. Evaluation of various cardiac biomarker shows involvement of cardiovascular system in $65.8 \%$ of the cases where at least one of the markers (Trop I, NT-pro BNP, CPK-MB) was elevated. 16 cases (20.2\%) required mechanical respiratory support out of which 15 cases (93.8\%) had evidence of cardiovascular involvement. Overall mortality was $8.9 \%$ (7 cases died) and all of them were having cardiovascular involvement. Involvement of cardiovascular system was associated with increase requirement for mechanical ventilator and high mortality (overall $13.5 \%$ with cardiovascular involvement and $15.4 \%$ in patients with PCVD).
\end{abstract}

Conclusions: Cardiovascular system involvement in hospitalized cases of swine flu is found to be very common and associated with high morbidity and mortality.

Keywords: Swine flu; Cardiovascular system; Trop I; NT-proBNP; CPK-MB; Cardiac markers; Electrocardiography

\section{Introduction}

Swine flu H1N1 is a novel strain of Influenza A virus that is evolved by genetic reassortment and its infection in human being can lead to varying degree of respiratory infections ranging from mild flu to severe and life-threatening pneumonia, bronchitis, acute respiratory distress syndrome with high mortality [1].

Epidemiologic studies have noted an increase in cardiovascular deaths during influenza epidemics indicating that cardiovascular complications of influenza infection, including exacerbation of heart failure, acute ischemic heart disease, and less often acute myocarditis, are important contributors to morbidity and mortality during influenza infection. This effect was observed in both genders at all age groups [2].

A study in the United States has previously estimated that influenza causes up to 92000 deaths per year by triggering Acute Myocardial
Infarction (AMI) [3]. The first 3-5 days within influenza infection is the highest risk time for AMI and it may be missed as findings of dyspnea, chest pain, fever and leukocytosis are considered related to pneumonia alone [4]. Swine flu is primarily considered as respiratory system disease although there have been case reports of acute cardiovascular events during the course of swine flu [5-14]. Therefore this prospective study was planned to evaluate burden of cardiovascular involvement in hospitalized cases of swine flu and its significance on clinical course and outcome. We also evaluated impact of pre-existing cardiovascular disease (PCVD) on clinical course of swine flu.

\section{Materials and Methods}

This prospective cross-sectional study was carried out in the Department of Medicine, Sardar Patel Medical College \& Associated Group of Hospitals, Bikaner. There was a sudden increase in the cases 
of swine flu in the month of November 2018. Therefore, a new epidemic was anticipated and this study was planned. These epidemics was lasted till March 2019 and during this period a total of 447 cases were screened for $\mathrm{H} 1 \mathrm{~N} 1$ out of which 259 cases were found to be positive by RT-PCR and among them 79 were admitted in the dedicated swine flu ward and included in the study. Written informed consent was taken from all subjects or their legal guardians before enrolling for the study. Prior approval from Institutional Ethics Committee was taken,

\section{Inclusion criteria}

1. All patients of swine flu confirmed by positive RT-PCR admitted in the hospital.

2. Age $>18$ years.

\section{Exclusion criteria}

1. Patients having influenza infection other than swine flu.

2. Patients not giving informed consent.

Diagnosis of swine flu was confirmed by RT-PCR. All patients were evaluated as per proforma. Clinical and epidemiological data were collected. All patients were subjected for laboratory examination including complete blood count, renal function test, liver function test, blood sugar, CRP titre, ABG and chest $\mathrm{x}$-ray. Cardiovascular involvement was evaluated by clinical history and examination, Standard 12 lead ECG, chest x-ray, CPK-MB, NT-proBNP and Troponin I. CRP titer was measured by Turbidomatric method (normal value $0-5 \mathrm{mg} / \mathrm{L}$ ). CPK-MB was measured by Auto Analyzer (normal range 0-24U/L). Measurement of High sensitive Troponin I (Q) was done by Fully Automated Enzyme Linked Fluorescent assay (ELFA) which had cut off value $<19 \mathrm{ng} / \mathrm{L}$ (the upper reference limit; $99^{\text {th }}$ percentile in a healthy reference population). NT-proBNP was measured by ELFA with cut off value $300 \mathrm{pg} / \mathrm{ml}$. All patients were treated as per WHO guidelines [15] and followed-up during hospital stay.

Severity of swine flu was categorized as follow (according to Ministry of Health \& Family Welfare, Govt of India guidelines revised on 18/10/2016):

Category-A: Patients with mild fever plus cough / sore throat with or without bodyache, headache, diarrhoea and vomiting.

Category-B1: In addition to all the signs and symptoms mentioned under Category-A, these patients has high grade fever and severe sore throat.

Category-B2: In addition to all the signs and symptoms mentioned under Category-A, individuals having one or more of the following high risk conditions (i) Children with mild illness but with predisposing risk factors (ii) Pregnant women (iii) Persons aged 65 years or older (iv) Patients with lung diseases, heart disease, liver disease kidney disease, blood disorders, diabetes, neurological disorders, cancer and HIV/AIDS (v) Patients on long term cortisone therapy.

Category-C: In addition to the above signs and symptoms of Category- $\mathrm{A}$ and $\mathrm{B}$, if the patient has one or more of the following: Breathlessness, chest pain, drowsiness, fall in blood pressure, sputum mixed with blood, bluish discoloration of nails and worsening of underlying chronic conditions.

\section{Statistical analysis}

Anonymised data were analysed using SPSS software version 17.0. Chi square test, ANOVA test, Pearson's Correlation test, Linear Regression Analysis and Student ' $\mathrm{t}$ ' test were used for continuous variables.

\section{Results}

A total of 79 cases were studied out of which 35 (44.3\%) were males (mean age $52.89 \pm 17.7$ ranging $19-92$ years) and $44(55.7 \%)$ females (mean age $47.36 \pm 16.79$ ranging 21 to 79 years). Most of the females belonged to age group 18-35 years while most males belonged to age group $>55$ years. Maximum cases belonged to category C (49 Cases; 62\%) followed by 15 cases each in category B1 and B2. 13 cases $(16.46 \%)$ had one or more underlying pre-existing cardiovascular disease (PCVD) which included 12 cases of congestive heart failure (CHF), 11 Ischemic Heart Disease (IHD), 4 Dilated Cardiomyopathy, 3 hypertension, 2 atrial fibrillation (AF) and one case Rheumatic Heart Disease (RHD).

Cardiovascular manifestations at the time of admission are shown in table 1. Most common cardiovascular symptom was breathlessness (72.2\%) followed by chest pain (28.8\%), palpitation (16.5\%), presyncope $(10.1 \%)$, syncope (7.6\%) and giddiness (5.1\%). Pedal edema was present in $20.3 \%$ cases followed by tachycardia (19\%), raised JVP (11.4\%), murmur (7.6\%), irregularly irregular pulse (4\%) and bradycardia and hypotension in $2.5 \%$ cases each.

ECG manifestations are shown in table 2. Most common ECG manifestation was raised QTc interval.

Evaluation of various cardiac biomarker shows involvement of cardiovascular system in $65.8 \%$ (52 patients) of the cases where at least one of the markers (Trop I, NT-pro BNP, CPK-MB) was elevated. All the markers were elevated in 4 cases, 25 cases had elevated both Trop-I and NT-proBNP, 18 only NT-pro BNP, 3only Trop I and 2 cases had only elevated CPK MB.

Overall Trop I was elevated in 32 cases (40.5\%) out of which 26 (81.3\%) belonged to category C. Trop I was mildly ( $>19$ to 190$)$ elevated in 19 cases, $191-2000$ in 9 and highly elevated $(>2000)$ in 4 cases. It was elevated in all the cases with STMI. 11 (84.6\%) cases with PCVD had elevated Trop-I while it was raised in $31.8 \%$ cases without PCVD. It was significantly elevated in patients presenting with chest pain $(72.7 \%$ of the cases who presented with chest pain, $\mathrm{p}<0.001)$, breathlessness $(50.9 \%, \mathrm{p}<0.003)$, palpitation $(76.9 \%$,

Table 1: Cardiovascular manifestations in cases of swine flu-clinical presentations.

\begin{tabular}{|l|c|c|c|}
\hline \multicolumn{1}{|c|}{$\begin{array}{c}\text { Symptoms and } \\
\text { Signs }\end{array}$} & \multicolumn{3}{|c|}{ No. of Cases (\%) } \\
\cline { 2 - 4 } Breathlessness & $29(82.9)$ & $28(63.6)$ & $57(72.2)$ \\
\hline Chest Pain & $10(28.6)$ & $12(27.3)$ & $22(27.8)$ \\
\hline Palpitation & $6(17.1)$ & $7(15.9)$ & $13(16.5)$ \\
\hline Pre Syncope & $5(14.3)$ & $3(6.8)$ & $8(10.1)$ \\
\hline Syncope & $2(5.7)$ & $4(9.1)$ & $6(7.6)$ \\
\hline Giddiness & $1(2.9)$ & $3(6.8)$ & $4(5.1)$ \\
\hline Tachycardia & $6(17.1)$ & $9(20.5)$ & $15(19.0)$ \\
\hline Bradycardia & $1(2.9)$ & $1(2.3)$ & $2(2.5)$ \\
\hline Pedal Edema & $8(22.9)$ & $8(18.2)$ & $16(20.3)$ \\
\hline JVP Raised & $5(14.3)$ & $4(9.1)$ & $9(11.4)$ \\
\hline Murmur & $6(17.1)$ & 0 & $6(7.6)$ \\
\hline Irregularly & $1(2.9)$ & $2(5.0)$ & $3(4.0)$ \\
\hline Irregular Pulse & $2(5.7)$ & 0 & $2(2.5)$ \\
\hline Hypotension & & & Total (No=79) \\
\hline
\end{tabular}


Table 2: ECG manifestations in cases of swine flu.

\begin{tabular}{|l|c|c|c|c|c|c|}
\hline \multirow{2}{*}{ ECG Findings } & \multicolumn{2}{|c|}{ PCVD $(\mathbf{n = 1 3 )}$} & \multicolumn{2}{c|}{ No PCVD (n=66) } & \multicolumn{2}{c|}{ Total $(\mathbf{n}=\mathbf{7 9 )}$} \\
\cline { 2 - 7 } & No. & $\%$ & No. & $\%$ & No & $\%$ \\
\hline Atrial Fibrillation & 3 & 23.1 & 0 & - & 3 & 3.8 \\
\hline STEMI & 2 & 15.4 & 2 & 3.0 & 4 & 5.1 \\
\hline RBBB & 1 & 7.7 & 3 & 4.5 & 4 & 5.1 \\
\hline LBBB & 2 & 15.4 & 0 & - & 2 & 2.5 \\
\hline P. Mitrale & 2 & 15.4 & 1 & 1.5 & 3 & 3.8 \\
\hline P. Pulmonale & 1 & 7.7 & 1 & 1.5 & 2 & 2.5 \\
\hline T-wave Inversion & 0 & - & 5 & 7.6 & 5 & 6.3 \\
\hline Type 1 AV Block & 0 & - & 1 & 1.5 & 1 & 1.3 \\
\hline $\begin{array}{l}\text { QTC Interval } \\
\text { (>0.44 sec) }\end{array}$ & 7 & 53.8 & 14 & 21.2 & 21 & 26.6 \\
\hline Sinus Bradycardia & 0 & - & 2 & 3.0 & 2 & 2.5 \\
\hline Sinus Tachycardia & 1 & 7.7 & 14 & 21.2 & 15 & 19.0 \\
\hline NSR & 4 & 30.8 & 37 & 56.1 & 41 & 51.9 \\
\hline
\end{tabular}

$\mathrm{p}<0.005)$ and syncope or presyncope $(71.4 \%, \mathrm{p}=0.036)$. Distribution of Trop-I level is shown in table 3.

NT-proBNP was raised in 47 (59.5\%) cases; all the 13 cases with PCVD had elevated NT-proBNP. Elevated NT-proBNP was significantly correlated with presenting symptoms like chest pain $(\mathrm{p}=0.046)$, breathlessness $(\mathrm{p}=0.037)$, palpitation $(\mathrm{p}=0.044)$, syncope or presyncope $(\mathrm{p}=0.036)$.It was also significantly correlated with CRP titre $(\mathrm{p}=0.003)$, atrial fibrillation $(\mathrm{p}=0.032)$, STMI $(\mathrm{p}=0.013)$ and prolonged QTc interval $(\mathrm{p}<0.001)$.Distribution of NT-proBNP level is shown in table 4.

CPK-MB was raised in 7 (7.6\%) cases; one with PCVD while 6 did not have any PCVD. 3 cases had ST segment elevation. Duration of illness was 6 days in 1 case, 5 days and 4 days in 3 cases each.

CRP titre was raised $(>5 \mathrm{mg} / \mathrm{L})$ in $98.1 \%$ of the cases with cardiovascular involvement and in $62.9 \%$ of the cases without cardiovascular involvement.

In our study 16 cases (20.2\%) out of 79 required mechanical respiratory support (invasive or non-invasive) out of which 15 (93.8\%) were having raised levels of any one of the cardiac biomarker (Troponin I, CPK-MB, NT-proBNP) suggestive of increased requirement of ventilator support in patients who were having cardiovascular involvement.

Overall mortality in our cases was $8.9 \%$ ( 7 cases died), all of them were having cardiovascular involvement. Involvement of cardiovascular system was associated with high mortality (overall $13.5 \%$ with cardiovascular involvement and in patients with PCVD 15.4\%) (Table 5). Pearson's Correlation analysis (Graph 1) and Multiple Linear Regression Analysis (ANOVA) (Graph 2) in relation to outcome also show that involvement of cardiovascular system was associated with poor outcome.

\section{Discussion}

We found significant number of the cases of swine flu $(74.7 \%)$ presented with symptoms suggestive of cardiovascular involvement (72.2\% breathlessness, $27.8 \%$ chest pain, $16.5 \%$ palpitation, $10.1 \%$ presyncope, $7.6 \%$ syncope and $5.1 \%$ giddiness) at the time of hospitalization. We did not find any study evaluating cardiovascular
Table 3: Distribution of Trop I in relation to different parameters.

\begin{tabular}{|c|c|c|c|c|c|c|}
\hline \multirow{2}{*}{\multicolumn{2}{|c|}{ Parameter }} & \multicolumn{2}{|c|}{$\begin{array}{l}\text { Raised Trop I } \\
\qquad(\mathrm{N}=32)\end{array}$} & \multicolumn{2}{|c|}{$\begin{array}{l}\text { Normal Trop I } \\
(\mathrm{N}=47)\end{array}$} & \multirow[t]{2}{*}{$\mathbf{p}$} \\
\hline & & No. & $\%$ & No & $\%$ & \\
\hline \multirow{2}{*}{ Age } & \multirow{2}{*}{\begin{tabular}{|l|} 
Mean \\
Range
\end{tabular}} & \multicolumn{2}{|c|}{$57.9 \pm 17.4$} & \multicolumn{2}{|c|}{$44.2 \pm 15.77$} & \multirow{2}{*}{$<0.001^{*}$} \\
\hline & & \multicolumn{2}{|c|}{$21-92$ years } & \multicolumn{2}{|c|}{$46-75$ years } & \\
\hline \multirow{2}{*}{ Sex } & Male & 18 & 56.3 & 17 & 36.2 & \multirow{2}{*}{0.078} \\
\hline & Female & 14 & 43.7 & 30 & 63.8 & \\
\hline \multirow{2}{*}{$\begin{array}{l}\text { Duration of } \\
\text { Illness }\end{array}$} & Mean & \multicolumn{2}{|c|}{$3.81 \pm 1.74$} & \multicolumn{2}{|c|}{$3.49 \pm 1.06$} & \multirow{2}{*}{0.310} \\
\hline & Range & & ays & & days & \\
\hline \multirow{2}{*}{$\begin{array}{l}\text { Duration of } \\
\text { Hospital Stay }\end{array}$} & Mean & \multicolumn{2}{|c|}{$8.2 \pm 3.01$} & \multicolumn{2}{|c|}{$7.06 \pm 2.2$} & \multirow{2}{*}{0.059} \\
\hline & Range & \multicolumn{2}{|c|}{ 3-19 days } & \multicolumn{2}{|c|}{$6-14$ days } & \\
\hline \multicolumn{2}{|l|}{ Chest pain } & 16 & 72.7 & 6 & 27.3 & $<0.001^{*}$ \\
\hline \multicolumn{2}{|c|}{ Breathlessness } & 29 & 50.9 & 28 & 49.1 & $0.003^{*}$ \\
\hline \multicolumn{2}{|l|}{ Palpitation } & 10 & 76.9 & 3 & 23.1 & $0.005^{*}$ \\
\hline \multicolumn{2}{|c|}{ Syncope/Presyncope } & 10 & 71.4 & 4 & 28.6 & $0.036^{*}$ \\
\hline \multicolumn{2}{|c|}{ Cardiomegaly } & 9 & 28.1 & 4 & 8.5 & $0.021^{*}$ \\
\hline \multicolumn{2}{|l|}{ PCVD } & 10 & 31.3 & 3 & 6.4 & $0.003^{*}$ \\
\hline \multicolumn{2}{|l|}{ STEMI } & 4 & 100 & 0 & - & $0.013^{*}$ \\
\hline \multirow{3}{*}{$\begin{array}{l}\text { Category of } \\
\text { swine flu }\end{array}$} & C & 26 & 81.3 & 22 & 46.8 & \multirow{3}{*}{$0.006 *$} \\
\hline & B2 & 4 & 18.8 & 11 & 23.4 & \\
\hline & B1 & 2 & 6.3 & 14 & 29.8 & \\
\hline \multicolumn{2}{|c|}{$\begin{array}{l}\text { Ventilator support } \\
\text { required }\end{array}$} & 12 & 75 & 4 & 25 & $0.002 *$ \\
\hline \multirow{2}{*}{ Outcome } & Stable & 25 & 78.1 & 47 & 100 & * * \\
\hline & Death & 7 & 21.9 & 0 & 0 & 0.001 \\
\hline
\end{tabular}

*Statistically significant

symptoms at the time of presentation in cases of swine flu although previous study on clinical profile has shown breathlessness in $29.54 \%$ to $83.1 \%$ cases, chest pain in $13.3 \%-49.2 \%$ and syncope in $6.7 \%$ [1619]. Thus our study suggests that evaluation for sign and symptoms of cardiovascular symptoms should be done at the time of presentation as they are often overlooked because of focus on respiratory symptoms like rhinorrhea, cold symptoms, throat pain and cough and patients also often ignore symptoms related to the cardiovascular system.

Most common ECG change observed in our study was prolongation of QTc interval which was observed in $21(26.6 \%)$ cases out of which 7 were already having documented QTc prolongation due to underlying PCVD and 14 cases developed QTc prolongation before the start of treatment at the time of hospitalization therefore probably because of underlying swine flu infection. We also observed AF, RBBB, LBBB and type I AV block. In literature we found some case reports on rhythm disorders in swine flu like VF, AF, complete heart block, first degree heart block, bradycardia and wide complex tachycardia $[13,14,16,20]$. Although precise mechanisms by which ECG manifestations are produced in H1N1 influenza are still unknown it may be a direct cytolytic effect of virus on myocardial cells with cytotoxic immunologic interaction, ischemia due to intracapillary thrombosis, inflammatory edema pressing $\mathrm{AV}$ node and other conduction tissue or primarily affecting conduction tissue/AV nodal pacemaker cells resulting in resting membrane potential changes [13,21,22].

In our study 4 patients developed STEMI, 2 of them were known case of pre-existing CAD and 2 were not (one 60 year old male and the other 64 year female), the values of Troponin I, NT-proBNP and CPK-MB in these 2 new cases was highly raised (778.2/7164/8.94 and $190.9 / 7016 / 6.3$ respectively). It has been shown that influenza may precipitate MI $[20,22,23]$. It has also been observed that incidence 
Table 4: Distribution of NT-proBNP in relation to different parameters.

\begin{tabular}{|c|c|c|c|c|c|c|}
\hline \multicolumn{2}{|c|}{ Parameter } & \multicolumn{2}{|c|}{$\begin{array}{l}\text { Pro BNP Normal } \\
(\mathrm{N}=32)\end{array}$} & \multicolumn{2}{|c|}{$\begin{array}{l}\text { Pro BNP Abnormal } \\
\qquad(\mathrm{N}=47)\end{array}$} & \multirow{4}{*}{$0.046^{*}$} \\
\hline & & \multirow{3}{*}{$\begin{array}{c}\text { No. } \\
27 \\
5\end{array}$} & \multirow{2}{*}{$\begin{array}{c}\% \\
84.4\end{array}$} & \multirow{2}{*}{$\begin{array}{l}\text { No } \\
30\end{array}$} & \multirow{2}{*}{$\begin{array}{c}\% \\
63.8\end{array}$} & \\
\hline Chest pain & No & & & & & \\
\hline Cnest pain & Yes & & 15.6 & 17 & 36.2 & \\
\hline \multirow{2}{*}{ Breathlessness } & No & 13 & 40.6 & 9 & 19.1 & \multirow{2}{*}{$0.037^{*}$} \\
\hline & Yes & 19 & 59.4 & 38 & 80.9 & \\
\hline \multirow{2}{*}{ Palpitation } & No & 30 & 93.8 & 36 & 76.6 & \multirow{2}{*}{$0.044^{*}$} \\
\hline & Yes & 2 & 6.3 & 11 & 23.4 & \\
\hline \multirow{2}{*}{ Syncope } & No & 32 & 100 & 41 & 87.2 & \multirow{2}{*}{$0.036 *$} \\
\hline & Yes & 0 & - & 6 & 12.8 & \\
\hline \multirow{2}{*}{ Pre-Syncope } & No & 31 & 96.9 & 40 & 85.1 & \multirow{2}{*}{0.089} \\
\hline & Yes & 1 & 3.1 & 7 & 14.9 & \\
\hline \multirow{2}{*}{ PCVD } & No & 32 & 100 & 34 & 72.3 & \multirow{2}{*}{$0.001^{*}$} \\
\hline & Yes & 0 & - & 13 & 27.7 & \\
\hline \multirow{2}{*}{ Pregnant } & No & 26 & 81.3 & 45 & 95.7 & \multirow{2}{*}{$0.036^{*}$} \\
\hline & Yes & 6 & 18.8 & 2 & 4.3 & \\
\hline \multirow{2}{*}{ Tachycardia } & No & 28 & 87.5 & 37 & 88.7 & \multirow{2}{*}{0.593} \\
\hline & Yes & 4 & 12.5 & 10 & 21.3 & \\
\hline \multirow{2}{*}{ Raised JVP } & No & 32 & 100 & 38 & 80.9 & \multirow{2}{*}{$0.009 *$} \\
\hline & Yes & 0 & - & 9 & 19.1 & \\
\hline \multirow{4}{*}{$\mathrm{PaO}_{2} / \mathrm{FiO}_{2}$} & $>300$ & 12 & 37.5 & 11 & 23.4 & \multirow{4}{*}{0.458} \\
\hline & $200-300$ & 7 & 21.9 & 12 & 25.5 & \\
\hline & $100-200$ & 9 & 28.1 & 13 & 27.7 & \\
\hline & $<100$ & 4 & 12.5 & 11 & 23.4 & \\
\hline QTc Interval & Total cases=21 & 2 & 9.5 & 19 & 90.5 & $0.001^{*}$ \\
\hline \multirow{2}{*}{ CRP Titre } & $>5$ & 23 & 71.9 & 45 & 95.7 & \multirow{2}{*}{$0.003^{*}$} \\
\hline & $\leq 5$ & 9 & 28.1 & 2 & 4.3 & \\
\hline \multirow{2}{*}{ CPK-MB } & $>24$ & 2 & 6.3 & 4 & 8.5 & 0710 \\
\hline & $\leq 24$ & 30 & 93.8 & 43 & 91.5 & 0.110 \\
\hline Tron 1 & Raised & 3 & 9.4 & 29 & 61.7 & $<0001 *$ \\
\hline Irop I & Normal & 29 & 90.6 & 18 & 38.3 & $<0.001$ \\
\hline Ventilation & No & 29 & 90.6 & 34 & 72.3 & $0 \Omega 47 *$ \\
\hline ventildion & Yes & 3 & 9.4 & 13 & 27.7 & 0.047 \\
\hline
\end{tabular}

*Statistically significant

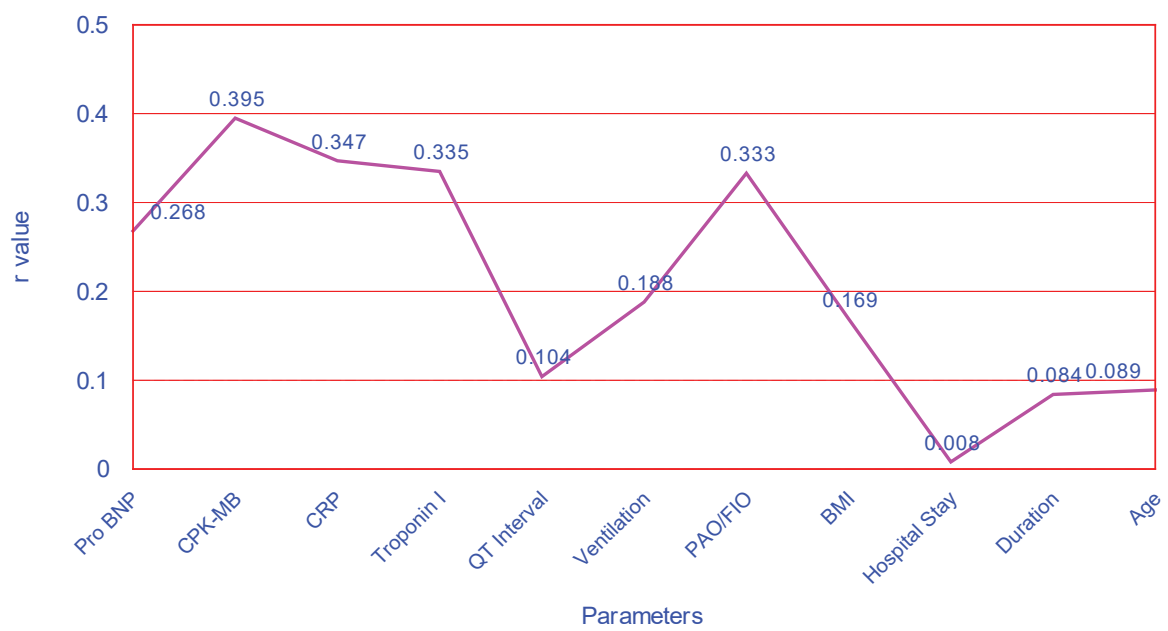

Graph 1: Pearson's Correlation according to outcome. 
Table 5: Cardiovascular parameters in relation to Outcome.

\begin{tabular}{|c|c|c|c|c|c|c|c|c|c|}
\hline \multirow{2}{*}{\multicolumn{3}{|c|}{ Parameters }} & \multirow{3}{*}{$\begin{array}{c}\text { Total } \\
2\end{array}$} & \multicolumn{2}{|c|}{ Dead } & \multicolumn{2}{|c|}{ Survive } & \multirow{2}{*}{$\chi^{2}$} & \multirow{2}{*}{ p } \\
\hline & & & & \multirow{2}{*}{$\begin{array}{c}\text { No. } \\
1\end{array}$} & \multirow{2}{*}{$\begin{array}{c}\% \\
50\end{array}$} & \multirow{2}{*}{$\begin{array}{c}\text { No. } \\
1\end{array}$} & \multirow{2}{*}{$\begin{array}{c}\% \\
50\end{array}$} & & \\
\hline \multirow{14}{*}{ ECG } & \multirow{3}{*}{ Heart Rate } & $<60$ & & & & & & \multirow{3}{*}{8.240} & \multirow{3}{*}{$0.016^{*}$} \\
\hline & & $60-100$ & 63 & 3 & 4.8 & 60 & 95.2 & & \\
\hline & & $>100$ & 14 & 3 & 21.4 & 11 & 78.6 & & \\
\hline & \multicolumn{2}{|c|}{ Atrial Fibrillation } & 3 & 1 & 33.3 & 2 & 66.7 & 2.313 & 0.128 \\
\hline & \multicolumn{2}{|l|}{ STEMI } & 4 & 0 & - & 4 & 100 & 0.410 & 0.522 \\
\hline & \multicolumn{2}{|l|}{ RBBB } & 4 & 1 & 25.0 & 3 & 75.0 & 1.359 & 0.244 \\
\hline & \multicolumn{2}{|c|}{ Sinus Tachycardia } & 15 & 3 & 20.0 & 12 & 80.0 & 2.845 & 0.092 \\
\hline & \multicolumn{2}{|c|}{ Sinus Bradycardia } & 2 & 1 & 50.0 & 1 & 50.0 & 4.300 & $0.038 *$ \\
\hline & \multicolumn{2}{|l|}{ LBBB } & 2 & 0 & - & 2 & 100 & 0.199 & 0.655 \\
\hline & \multicolumn{2}{|c|}{ P. Pulmonale } & 2 & 0 & - & 2 & 100 & 0.199 & 0.655 \\
\hline & \multicolumn{2}{|l|}{ P. Mitrale } & 3 & 0 & - & 3 & 100 & 0.303 & 0.582 \\
\hline & \multicolumn{2}{|c|}{ T-Wave Inversion } & 5 & 0 & - & 5 & 100 & 0.519 & 0.471 \\
\hline & \multicolumn{2}{|c|}{ ST Depression } & 1 & 0 & - & 1 & 100 & 0.098 & 0.754 \\
\hline & \multicolumn{2}{|c|}{ Type 1 AV Block } & 1 & 0 & - & 1 & 100 & 0.098 & 0.754 \\
\hline \multirow{3}{*}{ CRP Titre } & \multicolumn{2}{|l|}{ Normal } & 11 & 0 & - & 11 & 100 & \multirow{2}{*}{1.242} & \multirow{2}{*}{0.265} \\
\hline & Abnormal & & 68 & 7 & 10.3 & 61 & 89.7 & & \\
\hline & & Mean & & & .44 & & .88 & 3.244 & $0.002 *$ \\
\hline & Normal & & 73 & 4 & 5.5 & 69 & 94.5 & 12609 & ก $001 *$ \\
\hline CPK-MB & Abnormal & & 6 & 3 & 50.0 & 3 & 50.0 & 13.000 & $<0.001$ \\
\hline & & Mean & & & 90 & & .52 & 3.770 & $<0.001^{*}$ \\
\hline & $<19$ & & 47 & 0 & - & 47 & 100 & & \\
\hline & $19-190$ & & 19 & 4 & 21.1 & 15 & 78.9 & 16506 & ค คด1* \\
\hline Trop I & $191-2000$ & & 9 & 1 & 11.1 & 8 & 88.9 & 10.500 & 0.001 \\
\hline & $>2000$ & & 4 & 2 & 50.0 & 2 & 50.0 & & \\
\hline & & Mean & & 6179 & 33.99 & 380 & 2.24 & 3.118 & $0.003^{*}$ \\
\hline & Normal & & 47 & 0 & - & 47 & 100 & 11201 & ค००1* \\
\hline NT-proBNP & Abnormal & & 32 & 7 & 21.9 & 25 & 78.1 & 11.201 & 0.001 \\
\hline & Mean \pm SD & & & 6045 & 49.94 & 2063 & 93.02 & 2.444 & $0.017^{*}$ \\
\hline
\end{tabular}

*Statistically significant

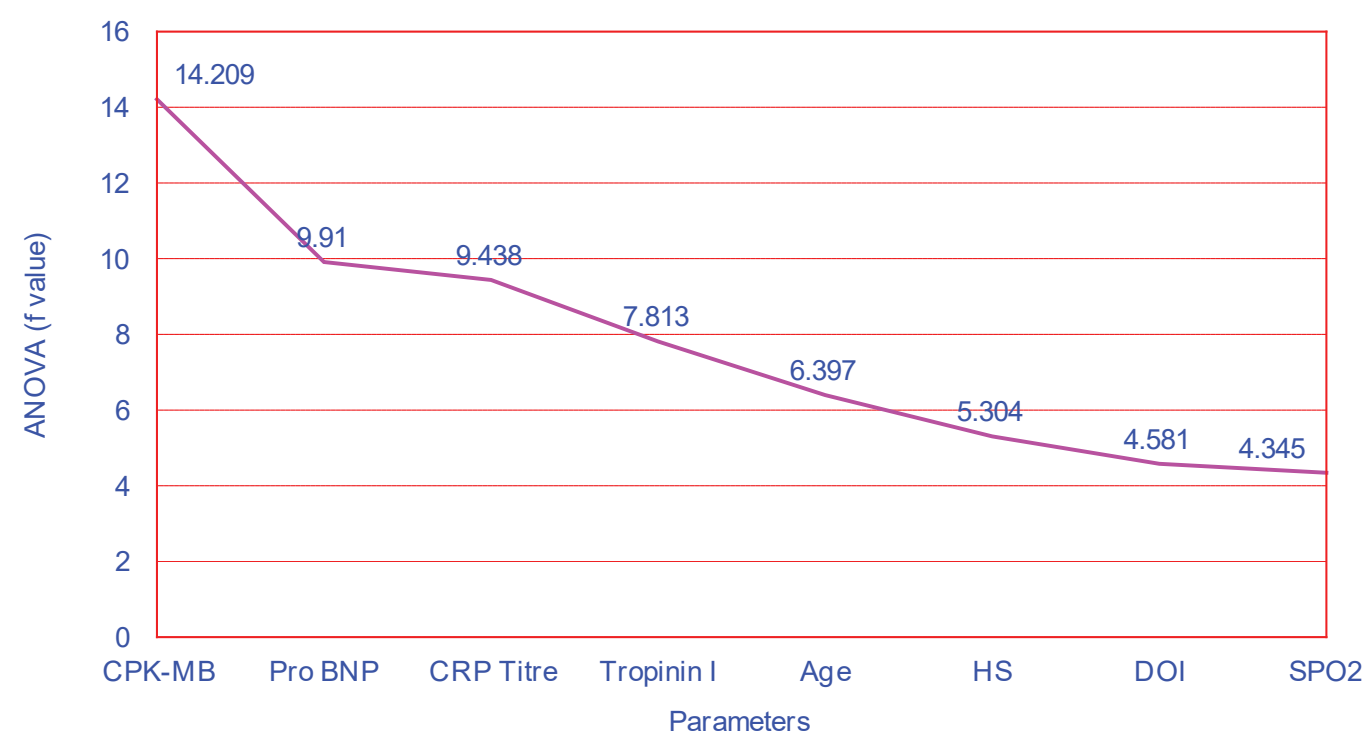

Graph 2: Multiple Linear Regression Analysis (ANOVA) in relation to outcome. 
of cardiovascular diseases like IHD, MI and $\mathrm{AF}$ are increased during influenza epidemic [3,24-26]. Acute MI shows a seasonal variation, having its highest incidence in the winter months $[3,24,27]$. Influenza activity has been suggested as a reason for this winter peak in the MI rate $[3,24,28]$. It is thought that influenza acts by many mechanisms, including inflammatory release of cytokines that causes a prothrombotic state, local disruption of coronary plaques, as well as physiological effects such as hypoxia and tachycardia, to cause acute obstruction of coronary arteries that may be otherwise subcritical stenosed [29]. Other mechanisms include sympathetic activation with subsequent effects on vascular tone with vasoconstriction; thrombogenesis through the nonspecific pro-coagulant and thrombophilic effects of inflammation; epithelial dysfunction; and inadequate coronary artery blood flow through increased metabolic demand with fever and tachycardia, reduced oxygen saturation and hypotension with secondary vasoconstriction [30]. Further, influenza has been shown to produce direct effects on the heart. Histopathological and molecular studies on influenza-infected mice have shown that the virus can be isolated from heart tissue and that its presence leads to local inflammatory changes [31].

Our study shows elevation of various cardiac markers in significant number of the cases $(65.8 \%)$ indicative of myocardial injury in swine flu and elevation of CRP titre in most of these cases suggest that it may be because of severe systemic inflammatory response leading to myocarditis. Although we did not find such studies on swine flu but it has been reported in cases of influenza by previous workers. Kaji M, et al. [32] in 2001 did a study on 96 confirmed cases of influenza and found $11.4 \%$ patients had evidence of myocardial injury by measuring serum myosin light chain I concentrations suggesting asymptomatic myocardial injury may be present in patients with influenza even when they have no symptoms suggestive of myocardial injury. Ison MG, et al. [33] in 2005 concluded that most ambulatory young adults with acute influenza have clinically insignificant abnormal electrocardiogram findings early during the illness which resolve promptly and are not associated with changes in cardiac markers or echocardiogram findings. The prevalence of fulminant myocarditis

Table 6: Pre-existing cardiovascular disease \& clinical course of swine flu.

\begin{tabular}{|c|c|c|c|c|c|c|c|}
\hline & & \multicolumn{2}{|c|}{ PCVD (n=13) } & \multicolumn{2}{|c|}{ No PCVD (n=66) } & \multirow{2}{*}{$x^{2}$} & \multirow{2}{*}{ p } \\
\hline & & No. & $\%$ & No. & $\%$ & & \\
\hline Age & Mean \pm SD & \multicolumn{2}{|c|}{$63.38 \pm 9.07$} & \multicolumn{2}{|c|}{$47.14 \pm 17.80$} & 3.199 & $0.002 *$ \\
\hline \multirow{2}{*}{ Sex } & Female & 4 & 30.8 & 40 & 60.6 & \multirow{2}{*}{3.918} & \multirow{2}{*}{$0.042 *$} \\
\hline & Male & 9 & 69.2 & 26 & 39.4 & & \\
\hline \multirow{3}{*}{ Category } & B1 & 0 & - & 16 & 24.2 & \multirow{3}{*}{6.746} & \multirow{3}{*}{$0.034 *$} \\
\hline & B2 & 1 & 7.7 & 14 & 21.2 & & \\
\hline & C & 12 & 92.3 & 36 & 54.5 & & \\
\hline \multicolumn{2}{|l|}{ Chest Pain } & 10 & 76.9 & 12 & 18.2 & 18.651 & $<0.001^{*}$ \\
\hline \multicolumn{2}{|c|}{ Breathlessness } & 13 & 100 & 44 & 66.7 & 6.006 & $0.014 *$ \\
\hline \multicolumn{2}{|c|}{ Palpitation } & 8 & 61.5 & 5 & 7.6 & 23.005 & $<0.001^{*}$ \\
\hline \multirow{11}{*}{ ECG } & AF & 3 & 23.1 & 0 & - & 15.832 & $<0.001^{*}$ \\
\hline & STEMI & 2 & 15.4 & 2 & 3.0 & 3.449 & 0.063 \\
\hline & RBBB & 1 & 7.7 & 3 & 4.5 & 0.224 & 0.636 \\
\hline & S Tachycardia & 1 & 7.7 & 14 & 21.2 & 1.291 & 0.256 \\
\hline & S Bradycardia & 0 & - & 2 & 3.0 & 0.404 & 0.525 \\
\hline & LBBB & 2 & 15.4 & 0 & - & 10.418 & $0.001 *$ \\
\hline & P Pulmonale & 1 & 7.7 & 1 & 1.5 & 1.679 & 0.195 \\
\hline & P Mitrale & 2 & 15.4 & 1 & 1.5 & 5.719 & $0.017^{*}$ \\
\hline & T-wave Inversion & 0 & - & 5 & 7.6 & 1.051 & 0.305 \\
\hline & ST Depression & 0 & - & 1 & 1.5 & 0.199 & 0.655 \\
\hline & Type 1 AV block & 0 & - & 1 & 1.5 & 0.199 & 0.655 \\
\hline \multicolumn{2}{|c|}{ Cardiomegaly } & 3 & 4.5 & 10 & 76.9 & 41.384 & $<0.001^{*}$ \\
\hline \multirow{2}{*}{ Outcome } & Death & 2 & 15.4 & 5 & 7.6 & \multirow{2}{*}{0.820} & \multirow{2}{*}{0.365} \\
\hline & Stable & 11 & 84.6 & 61 & 92.4 & & \\
\hline \multirow{3}{*}{ Trop-I } & $<19$ & 3 & 23.1 & 44 & 66.7 & \multirow{2}{*}{8.563} & \multirow{2}{*}{$0.003 *$} \\
\hline & $\geq 19$ & 10 & 76.9 & 22 & 33.3 & & \\
\hline & Mean \pm SD & \multicolumn{2}{|c|}{$4771.06 \pm 11779.49$} & \multicolumn{2}{|c|}{$131.22 \pm 462.17$} & 3.275 & $0.002 *$ \\
\hline CPK-MB & Mean \pm SD & \multicolumn{2}{|c|}{$23.57 \pm 38.42$} & \multicolumn{2}{|c|}{$18.46 \pm 26.00$} & 0.595 & 0.554 \\
\hline CRP Titre & Mean \pm SD & \multicolumn{2}{|c|}{$65.78 \pm 55.83$} & \multicolumn{2}{|c|}{$75.50 \pm 77.91$} & 0.428 & 0.670 \\
\hline & Mean $\pm S D$ & & .90 & 150 & 8.53 & 4.899 & $<0.001^{*}$ \\
\hline Pro BNP & $\leq 300$ & 0 & - & 32 & 48.5 & 10.594 & $0.001 *$ \\
\hline & $>300$ & 13 & 100 & 34 & 51.5 & 10.594 & 0.001 \\
\hline
\end{tabular}

*Statistically significant 
associated with $\mathrm{H} 1 \mathrm{~N} 1$ virus is unknown because of the lack of comprehensive screening, with only a handful of case reports $[9,13,34$ 41] and autopsy findings in the literature [42].

Elevation of cardiac markers in cases with PCVD suggestive of aggravation of underlying cardiovascular disease. Most of the cases of category $\mathrm{C}$ have raised Trop I as compared to category B2 shows that elevation of Trop I levels may be related to severity of swine flu. NTproBNP is a marker of heart failure and it was elevated in 47 cases including all 13 cases with PCVD. This explains that influenza virus may precipitate heart failure in such cases.

\section{Pre-existing cardiovascular disease $\&$ clinical course of swine flu (Table 6)}

A total of 13 cases $(16.4 \%)$ were having underlying one or more PCVD. Previous studies has also reported underlying cardiovascular disease in $4.4 \%$ to $7.89 \%$ [1,17-19]. Mean age of subjects with PCVD was $63.38 \pm 9.07$ years as compared to $47.14 \pm 17.8$ without PCVD. Duration of illness at the time of hospitalization was more in those with PCVD which may be probably because these patients could not have recognized symptoms of swine flu and could have attributed the same to underlying heart disease. NT-proBNP and Trop I along with CRP titre were highly elevated in patients with PCVD indicative of more severe inflammatory response and deterioration of cardiovascular disease during swine flu infection. These patients had higher morbidity, more requirement for mechanical ventilator, greater length of hospital stay and high mortality. An epidemiological association between influenza epidemic and cardiovascular mortality $[27,28,43,44]$ has also been demonstrated moreover, the influenza vaccine can protect against cardiovascular complications in people with pre-existing CAD [26] Lippi G, et al. [28] reported that swine flu virus may be able to affect the CVS via humoral, biological and biochemical mechanisms. In acute coronary syndromes it may be reasonable to consider any acute $\mathrm{H} 1 \mathrm{~N} 1$ infection as a precipitating factor that is final event in propelling predisposed individuals over a threshold that precipitates development of infarction in synergy with other well-known triggers. Macintyre, et al. did a case control study on 559 participants which concluded that recent influenza infection was an unrecognized comorbidity in almost $10 \%$ of hospitalized patients and the influenza vaccination was significantly protective of AMI [26].

\section{Impact of CV involvement in cases of swine flu who do not have PCVD}

Evaluation by various cardiac markers is suggestive of involvement of cardiovascular system in $59.1 \%$ of the cases who never had underlying cardiovascular disease before the onset of swine flu infection and out of them 5 died indicate its importance in determining outcome. Although swine flu is predominantly a respiratory system disease but we observed that cardiovascular system is also significantly involved. Al-Ammodi M, et al. reported association of myocarditis and Acute Coronary Injury with influenza virus and its possible mechanisms of atherogenesis [39]. Madjid M, et al. reported significant role for influenza infection in the development of atherosclerosis and triggering of its complications via pro-inflammatory prothrombotic effects [3].

\section{Conclusion}

Swine flu is primarily considered a respiratory disease but we found significant involvement of cardiovascular system in such cases. Involvement of cardiovascular system in swine flu is not only associated with high morbidity (prolonged clinical course and increased requirement for ventilator support) but also with poor outcome (high mortality). Patients with pre-existing cardiovascular disease ignore symptoms of swine flu in early stage therefore delaying in seeking medical attention and it is also associated with high morbidity and mortality. Due attention should be given to clinical symptomatology and evaluation with appropriate investigation regarding cardiovascular system to reduce morbidity and mortality in swine flu.

\section{Funding}

None

\section{Competing Interest}

None declared

\section{Ethical Approval}

A prior approval has been taken from the Institutional Ethics Committee to carry out this work, and an informed consent was obtained from the subjects enrolled in this study.

\section{References}

1. Gaikwad LL, Haralkar S (2014) Clinico-epidemiological profile of influenza A H1N1 cases admitted at a tertiary care institute of Western India. Int J Med Sci Public Health 3: 1258-1261.

2. Wiwanitkit V (2009) Heart failure in swine flu: a note. J Card Fail 15: 812 .

3. Madjid M, Naghavi M, Litovsky S, Casscells SW (2003) Influenza and cardiovascular disease: a new opportunity for prevention and the need for further studies. Circulation 108: 2730-2736.

4. Smeeth L, Thomas SL, Hall AJ, Hubbard R, Farrington P, et al. (2004) Risk of myocardial infarction and stroke after acute infection or vaccination. N Engl J Med 351: 2611-2618.

5. Penela D, Regueiro-Cueva A, Ortiz-Perez JT, Sitges M (2010) Myocarditis due to pandemic influenza A (H1N1). Rev Esp Cardiol 63: 1386-1387.

6. Martin SS, Hollingsworth CL, Norfolk SG, Wolfe CR, Hollingsworth JW (2010) Reversible cardiac dysfunction associated with pandemic2009-H1N1 influenza A. Chest 137: 1195-1197.

7. Cobas M, Abbo L, Santos M, Baccini-Jauregui C, Pham S (2010) Successful management of fulminant influenza A subtype H1N1 myocarditis. BMJ Case Rep.

8. Chacko B, Peter JV, Pichmuthu K, Ramakrishna K, Moorthy M, et al. (2012) Cardiac manifestations in patients with pandemic (H1N1) 2009 virus infection needing intensive care. J Crit Care; 27: 106.e1-106.e6.

9. Erden I, Erden EC, Ozhan H, Basar C (2011) Acute myocarditis mimicking acute myocardial infarction associated with pandemic 2009 (H1N1) influenza A virus. Cardiol J 18: 552-555.

10. Gokhroo RK, Barjaty HD, Bhawna K (2011) Cardiac conduction system affection in a case of swine flu. J Assoc Physicians India 59: 51-52.

11. Khouzam RN, Parizianu C, Hafiz AM, Chawla S, Schwartz R (2011) Fulminant myocarditis associated with novel H1N1 influenza $A$. Heart Lung 40: 566-568.

12. Ito N, Sato M, Momoi N, Aoyagi $Y$, Endo K, et al. (2015) Influenza A H1N1 pdm09-associated myocarditis during zanamivir therapy. Pediatr Int 57: 1172-1174.

13. Wang J, Xu H, Yang X, Zhao D, Liu S, et al. (2017) Cardiac complications associated with the influenza viruses A subtype H7N9 vs. pandemic H1N1 in critically ill patients under intensive care. Braz J Infect Dis 21: 12-18. 
14. Parmar T, Sen S (2018) Cardiac arrest as a complication of H1N1 infection: A case report from India. J Anesth Inten Care Med 5: 1-3.

15. WHO (2010) WHO guidelines for pharmacological management of pandemic influenza A (H1N1) 2009 and other influenza viruses. Part I Recommendations, WHO, Geneva, Switzerland.

16. Ukimura A, Izumi T, Matsumori A, Clinical Research Committee on Myocarditis Associated with 2009 Influenza A (H1N1) Pandemic in Japan organized by Japanese Circulation Society (2010). A National Survey on Myocarditis Associated With the 2009 Influenza A (H1N1) Pandemic in Japan. Circ J 74: 2193-2199.

17. Dhawale S, Jayant S (2016) Clinical profile, morbidity and mortality among swine flu (H1N1) infected patients: 2015 Gwalior, Madhya Pradesh Pandemic, India. Int J Adv Med 3: 324-327.

18. Prakash G (2013) Epidemiological and clinical profile of patients with swine flu (Influenza A, H1N1) attending Guru Govindsingh Government Hospital, Jamnagar, India. J Res Med Den Sci 1: 1-6.

19. Arbat S, Dave M, Niranjane V, Rahman I, Arbat A (2017) Analyzing the clinical profile of swine flu/influenza A H1N1 infection in central India: a retrospective study. Virusdisease 28: 33-38.

20. Ona MA, Bashari DR, Tharayil Z, Charlot A, Hoskins I, et al. (2012) A case of fatal fulminant myocarditis presenting as an acute STsegment elevation myocardial infarction and persistent ventricular tachyarrhythmia associated with influenza A (H1N1) virus in a previously healthy pregnant woman. Cardiology 123: 103-107.

21. Kodama M (2010) Influenza Myocarditis. Circ J 74: 2060-2061.

22. Iwanaga N, Nakamura S, Fukuda $\mathrm{Y}$, Takazono $\mathrm{T}$, Imamura $\mathrm{Y}$, et al. (2014) A fatal case of acute myocardial infarction following the improvement of influenza A (H1N1) pdm2009-related acute myocarditis. Intern Med 53: 2153-2157.

23. Kwong JC, Schwartz KL, Campitelli MA, Chung H, Crowcroft NS, et al. (2018) Acute Myocardial Infarction after Laboratory-Confirmed Influenza Infection. N Engl J Med 378: 345-353.

24. Lichenstein R, Magder LS, King RE, King JC Jr (2012) The relationship between influenza outbreaks and acute ischemic heart disease in Maryland residents over a 7-year period. J Infect Dis 206: 821-827.

25. Warren-Gash C, Hayward AC, Hemingway $H$, Denaxas $S$, Thomas SL, et al. (2012) Influenza infection and risk of acute myocardial infarction in England and Wales: a CALIBER self-controlled case series study. J Infect Dis 206: 1652-1659.

26. Maclntyre CR, Mahimbo A, Moa AM, Barnes M (2016) Influenza vaccine as a coronary intervention for prevention of myocardial infarction. Heart 102: 1953-1956.

27. Sheth T, Nair C, Muller J, Yusuf S (1999) Increased winter mortality from acute myocardial infarction and stroke: the effect of age. J Am Coll Cardiol 33: 1916-1919.

28. Lippi G, Franchini M, Favaloro EJ (2010) Influenza and cardiovascular disease: does swine-origin, 2009 H1N1 flu virus represent a risk factor, an acute trigger, or both? Semin Thromb Hemost 36: 49-58.
29. Corrales-Medina VF, Suh KN, Rose G, Chirinos JA, Doucette S, et al. (2011) Cardiac complications in patients with community-acquired pneumonia: a systematic review and meta-analysis of observational studies. PLoS Med 8: e1001048.

30. Corrales-Medina VF, Madjid M, Musher DM (2010) Role of acute infection in triggering acute coronary syndromes. Lancet Infect Dis 10: 83-92.

31. Fislova T, Gocnik M, Sladkova T, Durmanova V, Rajcani J, et al. (2009) Multiorgan distribution of human influenza $A$ virus strains observed in a mouse model. Arch Virol 154: 409-419.

32. Kaji M, Kuno H, Turu T, Sato Y, Oizumi K (2001) Elevated serum myosin light chain I in influenza patients. Intern Med 40: 594-597.

33. Ison MG, Campbell V, Rembold C, Dent J, Hayden FG (2005) Cardiac findings during uncomplicated acute influenza in ambulatory adults. Clin Infect Dis 40: 415-422.

34. Bratincsak A, El Said HG, Bradley JS, Shayan K, Grossfeld PD, et al. (2010) Fulminant myocarditis associated with pandemic H1N1 influenza A virus in children. J Am Coll Cardiol 55: 928-929.

35. Chan K, Meek D, Chakravorty I (2011) Unusual association of ST-T abnormalities, myocarditis and cardiomyopathy with H1N1 influenza in pregnancy: two case reports and review of the literature. J Med Case Reports 5: 314

36. Barbandi M, Cordero-Reyes A, Orrego CM, Torre-Amione G, Seethamraju H (2012) Case series of reversible acute cardiomyopathy associated with H1N1 influenza infection. Methodist Debakey Cardiovasc J 8: 42-45.

37. Cabral M, Brito MJ, Conde M, Oliveira M, Ferreira GC (2012) Fulminant myocarditis associated with pandemic H1N1 influenza A virus. Rev Port Cardiol 31: 517-520.

38. Cunha B, Syed U, Mickail N (2010) Fulminant fatal swine influenza (H1N1): myocarditis, myocardial infarction, or severe influenza pneumonia? Heart Lung 39: 453-458.

39. Al-Ammodi M, Rao K, Rao S, Brewer J, Maglski A, et al. (2010) Fulminant myocarditis due to H1N1 influenza. Circ Heart Fail 3: e7e9.

40. Pavlidis AN, Giannakopoulos AK, Danias PG, Manolis AJ (2010) A Case of Acute Myocarditis Caused by Pandemic (H1N1) 2009 Influenza Virus. J Formos Med Assoc 109: 857-858.

41. Fagnoul D, Pasquier P, Bodson L, Ortiz JA, Vincent JL, et al. (2013) Myocardial dysfunction during H1N1 influenza infection. J Crit Care 28: 321-327.

42. Novel Swine-Origin Influenza A (H1N1) Virus Investigation Team, Dawood FS, Jain S, Finelli L, Shaw MW, et al. (2009) Emergence of a novel swine-origin influenza $\mathrm{A}$ (H1N1) virus in humans. N Engl J Med 360: 2605-2615.

43. Mamas MA, Fraser D, Neyses L (2008) Cardiovascular manifestations associated with influenza virus infection. Int J Cardiol 130: 304-309.

44. Estabragh ZR, Mamas MA (2013) The cardiovascular manifestations of influenza: a systematic review. Int J Cardiol 167: 2397-2403. 